\title{
Proteome analysis - A novel approach to understand the pathogenesis of Type 1 diabetes mellitus
}

\author{
Allan E. Karlsen, Thomas Sparre, Karin Nielsen, \\ Jørn Nerup and Flemming Pociot* \\ Steno Diabetes Center, Gentofte, Denmark
}

Type 1 (insulin-dependent) diabetes mellitus (T1DM) is associated with a specific destruction of the insulin-producing beta-cells in the islets of Langerhans. Several factors, e.g. genetic, environmental and immunologial, may be involved in the etiology and pathogenesis of T1DM. Autoreactive Tand B-lymphocytes, together with macrophages infiltrate the islets during the pathogenesis, releasing a mixture of cytokines, demonstrated to be specifically toxic to the beta-cells within the islets. Our goal is to understand the molecular mechanisms responsible for the beta-cell specific toxicity enabling us to design novel intervention strategies in T1DM. The proteome approach allows us to get a detailed picture of the beta-cell proteins, which change expression level or are post-translationally modified in different in vitro and in vivo models of T1DM-associated beta-cell destruction. Combining the information obtained from this extended proteome approach, with that of genetic-, transcriptome- and candidategene approaches, we believe that it is possible to reach this goal.

\section{Introduction}

Diabetes mellitus represents a heterogeneous group of disorders. Some distinct diabetic phenotypes can be characterized in terms of specific etiology and/or pathogenesis, but in many cases overlapping phenotypic characteristics make etiological and pathogenetical classification difficult.

Type 1 (insulin-dependent) diabetes mellitus (T1DM) is characterized by absolute insulin deficiency, abrupt onset of symptoms, proneness to ketosis and depen-

* Address for correspondence: Flemming Pociot, Steno Diabetes Center, Niels Steensensvej 2, DK-2820 Gentofte, Denmark. Fax: +45 4443 7313; E-mail: fpoc@novo.dk. dency on exogenous insulin to sustain life. It is the most common form of diabetes among children and young adults in populations of Caucasoid origin, where the prevalence is approximately $0.4 \%$. T1DM is traditionally regarded as a disease of the childhood and adolescent period. The age-specific incidence pattern is similar worldwide within this age segment, although the worldwide incidence differs markedly for the age group up to 15 years. The disease is rare before 6 months of age, but the incidence increases up to puberty, and thereafter declines. The overall age-adjusted incidence of T1DM varies from $0.1 / 100.000$ per year in China and Venezuela to 36/100.000 per year in Sardinia and Finland [1]. An increasing incidence of T1DM for individuals $<15$ years of age has been observed in many countries. Studies have suggested that more than $50 \%$ of all T1DM cases develop after the age of 21 years, and that the cumulative incidence (up to age 80 years) may reach approximately $1 \%[2,3]$.

T1DM is the result of pancreatic beta-cell destruction. The islets of Langerhans containing the insulinproducing beta-cells are scattered throughout the pancreas and comprise only $1-2 \%$ of the total tissue. They contain in addition to the beta-cells, the 3 other endocrine cell-types, the glucagon-producing alphacells, the somatostatin producing delta-cells and the PPcells producing pancreatic polypeptide, with the alpha$(\sim 20 \%)$ and beta-cells $(\sim 70 \%)$ constituting the major part of a normal islet (Fig. 1). All the 4 endocrine cell-types are believed to differentiate from stem-cell progenitor cells through a common pathway, which late in this maturation process branches out into the specific cell-types. Since T1DM is caused by a specific destruction of the beta-cells in the islets, a major goal within T1DM research is to understand the mechanisms responsible for the beta-cell maturation and associated phenomena making the beta-cell specifically recognized and destroyed in T1DM.

The etiology of T1DM is not yet fully understood. It is believed that genetic factors are a major compo- 


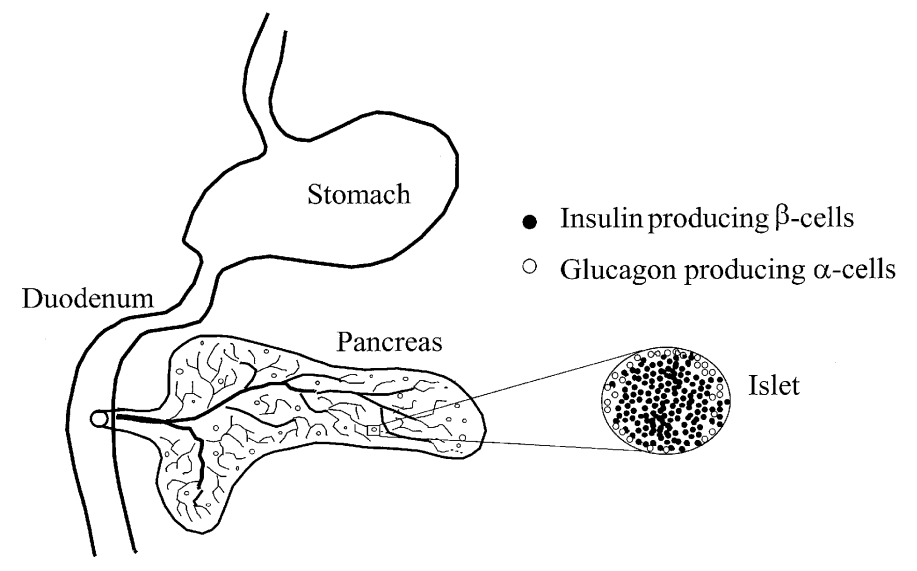

Fig. 1. The pancreatic islets of Langerhans.

Table 1

Candidate regions for Type 1 diabetes mellitus susceptibility genes identified through linkage analyses

\begin{tabular}{ll}
\hline Locus & Chromosome \\
\hline IDDM1 (HLA) & $6 \mathrm{p} 21$ \\
IDDM2 (INS) & $11 \mathrm{p} 15$ \\
IDDM3 & $15 \mathrm{q} 26$ \\
IDDM4 & $11 \mathrm{q} 13$ \\
IDDM5 & $6 \mathrm{q} 25$ \\
IDDM6 & $18 \mathrm{q}$ \\
IDDM7 & $2 \mathrm{q} 31$ \\
IDDM8 & $6 \mathrm{q} 25-\mathrm{q} 27$ \\
IDDM9 & $3 \mathrm{q} 21-\mathrm{q} 25$ \\
IDDM10 & $10 \mathrm{p} 11.2-\mathrm{q} 11.2$ \\
IDDM11 & $14 \mathrm{q} 24.3-\mathrm{q} 31$ \\
IDDM12 & $2 \mathrm{q} 33$ \\
IDDM13 & $2 \mathrm{q} 34$ \\
IDDM15 & $6 \mathrm{q} 21$ \\
IDDM17 & $10 \mathrm{q} 25$ \\
IDDM18 & $5 \mathrm{q} 33-\mathrm{q} 34$ \\
DXS1068 & Xq \\
GCK & $7 \mathrm{p}$ \\
\hline
\end{tabular}

nent in the etiology of T1DM. Genes within the MHC region on chromosome $6 \mathrm{p} 21$ comprise the major genetic locus determining predisposition to T1DM [46]. However, over the last 6-7 years extensive efforts have been invested in order to genetically map other chromosomal regions demonstrating evidence of linkage to T1DM [7-10]. This has led to the identification of at least 16 non-HLA loci demonstrating some evidence of linkage to T1DM (Table 1). Nevertheless, although large resources have been put into the genome scan approach, no evidence for any etiological mutation(s) has yet been obtained. We therefore believe that novel approaches or combinations of multidisciplinary approaches are needed to unravel the molecular basis of the disease. Combining genetic and proteomebased strategies may represent a valuable approach as discussed below. In addition to the well-established candidate gene and linkage approaches for identifying disease predisposing genes and proteins, methods for expression profiling have been developed over the last years. Expression profiling is the evaluation and comparison of expression levels of transcripts and/or proteins in diseased versus non-diseased tissues or in experimental model systems hereof.

Although the trigger(s) of T1DM is not yet identified, diet, environmental exposures and viral infections are putative etiologic agents involved in the pathogenesis. Most studies on diet components in the aetiology of T1DM have focused on the role of early exposure to bovine serum albumin in cow's milk [11]. Other dietary components may include nitrite and nitrates from food and drinking water [12]. Although a single major component of the diet still has to be demonstrated of importance in T1DM, studies of the increasing incidence rate in low-risk populations migrating to high-risk areas strongly suggest an environmental component in the disease etiology. Viruses, as an environmental factor, have been implicated in T1DM pathogenesis based on both case reports of virus isolation from pancreata of acutely diabetic deceased patients and induction of diabetes in animal models by infection with virus as well as on epidemiological studies examining recent-onset T1DM patients for virus-specific antibodies [13]. An etiological model reconciling these different aspects is shown in Fig. 2.

Research to elucidate the pathogenesis of T1DM has for many years mainly focused on the genetic and immnological markers associated with the disease and to a lesser degree on environmental factors. However, recently increasing focus has been directed towards the target cell, the beta-cells in the islets. Why are the beta- 


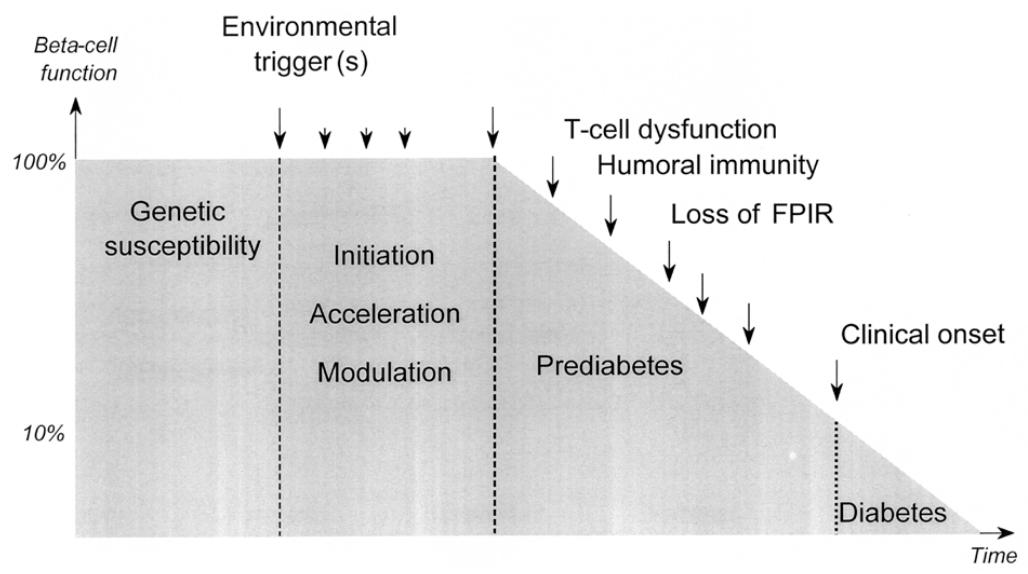

Fig. 2. Etiological model of Type 1 diabetes mellitus. If the total beta-cell mass in a healthy individual is set to be $100 \%$, some yet unrecognized environmental triggers may activate pathogenetic mechanisms leading to progressive loss of pancreatic islet beta-cells. This process is associated with a variety of immune phenomena, including infiltration of the islets with macrophages and auto-reactive T-lymphocytes (insulitis), and both T-cell (cell-mediated) and B-cell (humoral, antibody mediated) reactivity have been demonstrated prior to the onset of disease. However, the immunological mechanisms directly involved in beta-cell killing have not yet been clearly defined in man. Whereas only few diabetes-associated T-cell clones have been isolated, several autoantibodies to beta-cell proteins have been demonstrated up to several years before the onset of disease in blood from prediabetic and recent onset diabetic individuals. This autoimmune mediated destruction may progress slowly (over several years) or more rapid, dependent on the frequency and level of insults, influencing the balance between beta-cell destruction and regeneration in the individual islets. The end-point is the clinical onset of T1DM, when approximate $10 \%$ of the beta-cell mass remains. Until this time-point, the residual beta-cell mass is sufficient to maintain normal blood-glucose under normal conditions, however, the reduced beta-cell mass may become insufficient to challenges with higher demand for insulin secretion, as demonstrated by the loss of the first phase insulin response (FPIR).

cells specifically destroyed, when the other pancreatic endocrine cell-types of common origin in the islets, e.g. the alpha-cells, are not?

The original demonstration that cytokines [1416], especially interleukin-1- $\beta$ (IL-1 $\beta$ ) released from macrophages and T-lymphocytes during insulitis were specifically toxic to the beta-cells, enabled us in 1988 to construct a testable model of the pathogenesis of T1DM [17], the so-called "Copenhagen-model", outlined in details below and shown in its present form in Fig. 3.

The model predicts that anything from the external or internal environment which can destroy a beta-cell (nutrients? virus? chemicals? cytokines?) will lead to the release of beta-cell proteins. These proteins will be taken up by residing antigen presenting cells (APC; e.g. macrophages $(\mathrm{M} \varnothing)$ and dendritic cells) in the islets, will be processed to antigenic peptides and as such be presented by MHC-Class II molecules on the cell surface.

This activates the APCs to produce and secrete monokines (IL-1 $\beta$ and tumor necrosis factor (TNF) $\alpha$ and co-stimulatory signal(s)), which, if T-helper lymphocytes with receptors specifically recognizing the antigenic peptide are present in the islet, induce the transcription of a series of lymphokine genes.
One of these, interferon (IFN) $\gamma$, feedback stimulates the APC to increase expression of MHC-Class II molecules and IL-1 $\beta$ and $\mathrm{TNF} \alpha$. In addition, other cells of the APC lineage present in the islet are induced to secrete monokines. IL- $1 \beta$, potentiated by TNF $\alpha$ and IFN $\gamma$ is cytotoxic to beta-cells, primarily through the induction of free radical (FR: $\mathrm{NO}, \mathrm{O}_{2}$ ) formation in the islet. It has been confirmed by recent studies from several laboratories, including our own [18-24] that a role of FR is important, by demonstrating that nitric oxide (NO), and oxygen-derived FR are induced in islet cells by cytokines, and that the inducible nitric oxide synthase (iNOS), responsible for the induced NO production is induced by IL- $1 \beta$ in beta-cells (not present in other endocrine islet cells) from which we were able to clone it out [25]. In addition, also FR independent toxicity exists as shown in cytokine exposed human islets, where toxicity is still induced in the presence of NO-inhibitors as well as by combinations of cytokines, which do not induce FR production [26].

As part of the beta-cell destructive mechanism betacell proteins may be modified by the FR and in more antigenic forms presented to the immune system, thereby closing the loop in a self-perpetuating and selflimiting fashion. Thus, self-proteins presented by the MHC-Class-I molecules on the beta-cell or released during the beta-cell destruction by FR and taken up and 


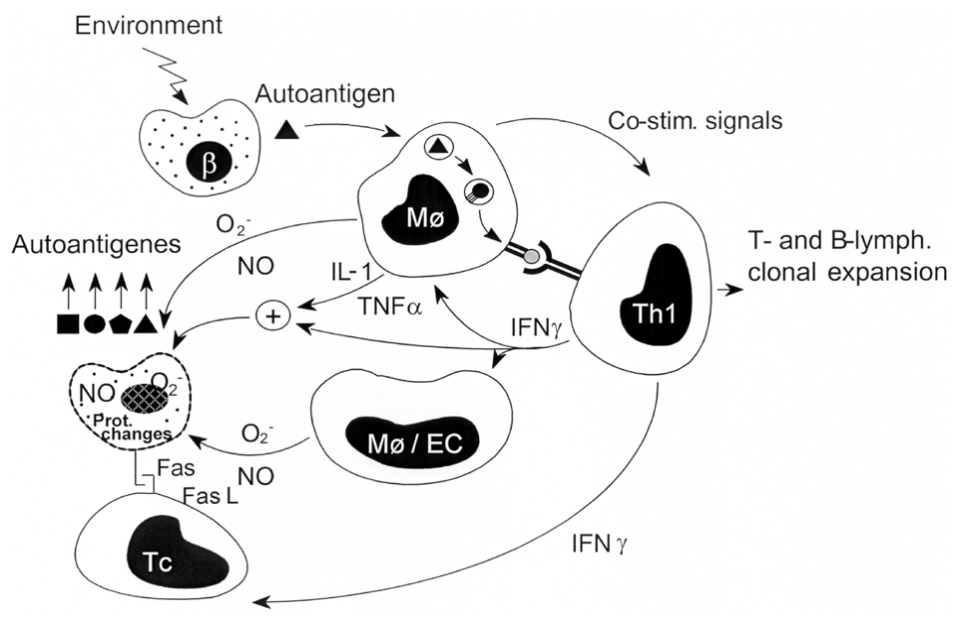

Fig. 3. Copenhagen model of beta-cell destruction.

presented by MHC-Class-II, need not be antigenic in their native conformation, but rather antigenecity may be a result of FR modification(s) [27]. This implies, that beta-cell proteins may become antigens when and because beta-cells are destroyed by cytokines. In addition the cytokine exposure has been shown to induce expression of FAS on the surface of the beta-cells. Interaction with the FAS-ligand on T-lymphocytes may therefore also represent a mechanism of induced betacell lysis/destruction [28,29].

The magnitude of beta-cell destruction will depend upon a) the velocity of the feed-back circuit between the APC and the T-helper lymphocyte, i.e. on the efficacy of antigen transport presentation/recognition, b) the magnitude and type of cytokine production, and c) on the capacity of beta-cell defense mechanisms during cytokine exposure [30]. The model has important implications, since it eliminates the need for the existence of specific environmental trigger(s), T1DM specific genes or gene mutations, and targeted exposure of beta-cells to the cytotoxic action of monokines.

Pro-inflammatory cytokines induce both protective and deleterious mechanisms in all cells with cytokine receptors. The resulting race between protective and deleterious mechanisms determines the outcome, which is whether the cell will die or survive. Indeed, both protective and deleterious mechanisms have been demonstrated in response to cytokines in different cell types, however, the response varies between cell types, and within a cell-type between species and probably also between humans with different genetic background. Thus, we hypothesize that in genetically predisposed individuals, T1DM develops when the deleterious events in the beta-cells prevail.
This would suggest that the pathogenesis of T1DM, i.e., the earliest events and mechanisms affecting the target cell, has two distinct phases: a non-antigen driven, non-lymphocyte dependent initiation phase, and an antigen driven (by multiple antigens?), lymphocyte dependent amplification and perpetuation phase.

The model further offers a possible explanation for the existing multitude of "autoantibodies" in the blood of newly diagnosed and prediabetic individuals (see below).

On this background we postulate that the remarkable specificity of cellular destruction in the islets in T1DM is due to an inherent vulnerability of beta-cells to cytokine-induced FR damage, acquired during its differentiation into the highly developed and metabolically sophisticated beta-cell. In other words, when exposed to proinflammatory cytokines the beta-cells die because they are beta-cells. Hence, detailed studies of the intracellular molecular events resulting from the interaction between immune effector molecules/cells and the target cell are in demand, and proteome analysis is a valuable tool to address this aspect.

\section{Antibody-markers of T1DM}

As mentioned above, T1DM is associated with the presence of one or more of a multitude of autoantobodies present in the blood of the majority of prediabetic and recent onset T1DM individuals. Whereas these antibody markers may show different degree of disease specificity [31,32], none of the known T1DMassociated autoantigens clearly qualifies for the position as the major or primary driving antigen responsi- 
ble for the amplification and perpetuation of beta-cell destruction. Rather they may serve as disease markers of a progressive beta-cell destruction. Nevertheless, proteome analysis of cytokine exposed islets/betacells may help identify new auto-antigen candidates among native (e.g., de novo synthesized or changed cellular localization) or FR-modified beta-cell specific proteins, to be tested for potential reactivity with antibodies present in the blood of prediabetic and recent onset diabetic individuals.

\section{Proteome analysis}

A cell is dependent upon a multitude of metabolic and regulatory pathways for survival. There is no strict linear relationship between the genes and the protein complement of a cell (i.e., mRNA levels may or may not correlate with the protein level) [33]. The ability of characterizing the protein expression profile in a cell or tissue at a given time point, reflecting the metabolic and functional status at that particular time point, is the hallmark of proteome analysis. Therefore, proteome technology is a suitable tool in the search for disease associated protein/protein pathways in T1DM and other diseases with a known target organ/cell. The possibility of comparing the status of the protein expression profile between healthy and diseased tissue/cells creates opportunities for new strategies to identify at the molecular level proteins and mechanisms involved in, or responsible for, disease progression. With focus on the beta-cell destruction associated with T1DM, this is discussed below.

\section{Proteome analysis in T1DM - an in vitro approach}

As detailed above, it is believed that T1DM is associated with the destructive effects of cytokines released in the islets during the insulitis process occurring before clinical onset of the disease. The Copenhagen model (Fig. 3) details this process, and is a testable model, which can be approached using proteome analysis as follows: Pancreatic islets of Langerhans are isolated, kept in culture in vitro and exposed to different cytokines for fixed periods of time. Extensive research has documented that the metabolic function of such isolated islets (from either rat, mouse and humans) may be severely impaired by the co-culture with cytokines, which dependent on combination and concentration, may also induce cell death [29]. Using this in vitro culture system with rat islets allowed us to make the first proteome analysis of cytokine-exposed islets [34-37].

Figure 4 illustrates the experimental design for these studies. First, isolated islets (from rat, mouse or human organ donors) are cultured in the absence or presence of cytokines for a set period of time (e.g. $24 \mathrm{~h}$, or ideally, for several different time-points to allow analysis of the kinetics of changes in protein expression). Different strategies may be applied in order to subsequently identify and quantify the different proteins (e.g. radiolabelling, labelling with fluorescent dyes or silver staining), each method with its pros and cons. We have used 35-S labelled methionine to radiolabel the proteins for $4 \mathrm{~h}$ at the end of the cytokine exposure period. The next step in the procedure is the separation of the proteins from the control and cytokine exposed islets, respectively, by two-dimensional electrophoresis (2DGE) according to charge (pI) and size (Mw) by isoelectrical focusing (IEF) or non-equilibrium $\mathrm{pH}$ gradient electrophoresis (NEPHGE) and SDS-page gel electrophoresis, respectively. The individual proteins may now be visualized using a phosphoimager (or on a $\mathrm{X}$-ray film) based on the incorporation of the 35-S labeled methionine, allowing quantification of the spots over a large dynamic range. An important point in the experimental design is to reduce the number of type 2 errors, e.g. minor irrelevant changes in protein expression, which may be caused by the experimental procedure as such. Therefore, it is essential to perform a number of independent experiments with islets material obtained from different batches of islets preparations (Panel 2, Fig. 4). This strategy is evident when thinking of analyses using human islets isolated from persons with different genetic background, where results obtained from one individual islet isolation, in addition to inherent influence of the genetics, may be influenced by variations in the tissue procurement and experimental setup, and therefore may not be reproducible in future experiments. Unfortunately, this necessary analysis of several gels from each group to be compared against each other represents a bottleneck in the experimental design. Whereas computer assisted alignment of the protein spots between the gels may be performed using different available software, even the most optimized 2DGE system will not consistently run gels $100 \%$ identical. Hence, careful manual evaluation and completion of the computer mediated alignment of the individual gels within the different experimental groups and between different groups is necessary, why a composite image is created and the different gels 


\section{1) In vitro labeling and 2D-analysis}
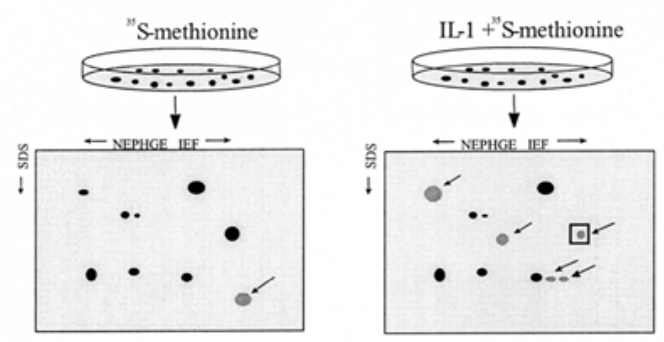

3) MS assisted protein identification

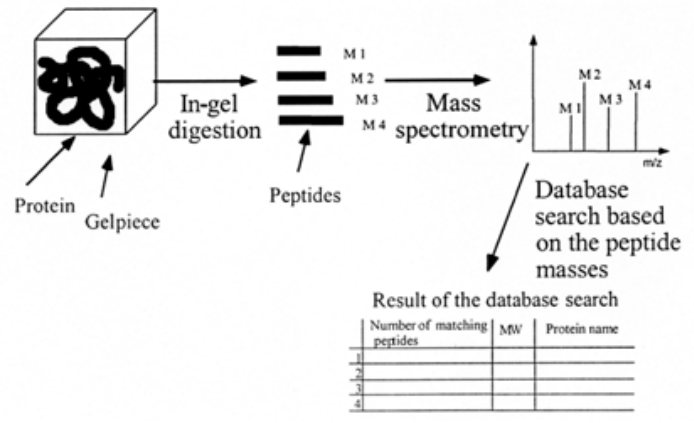

\section{2) Computer assisted 2D-gel analysis}

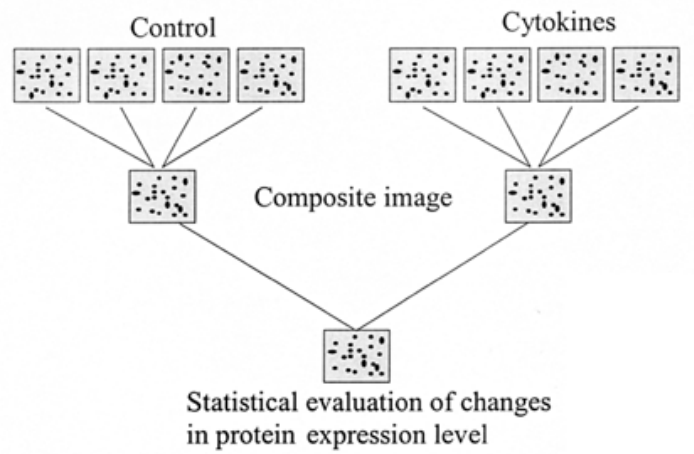

\section{4) Bioinformatics}

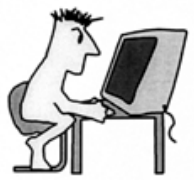

\section{5) Functional and/or genetic characterization}

Fig. 4. Experimental design for proteome analysis in Type 1 diabetes.

aligned to this. Having done that, it is possible to make statistical evaluation of the expression levels of the different proteins to identify those, which significantly change expression level in response to e.g. cytokine treatment (Panel 2, Fig. 4). The next step is to identify the proteins in the spots of changed expression level. This is effectively done by mass spectrometry (MS), as illustrated in Panel 3 (Fig. 4). The individual protein spots are excised from preparative gels, rehydrated and digested by a protease, e.g. trypsin, in situ in the gel plug. Following up-concentration of the material, it is subjected to MS-analysis and the resulting MS-profile analyzed in silico against all the theoretical MS-profiles calculated from available amino acid sequences present in public sequence databases. Based on this analysis and the available information of $\mathrm{Mw}$ and $\mathrm{pI}$ from the gel, the protein may be identified by its "fingerprint" if its sequence is present in the sequence databases. With completion of the human genome project, the success rate of identifying human proteins by mass spectrometry is now very high.

Knowing the identity of all the proteins of changed expression levels, the next task is to clarify how these proteins may be involved in cytokine-mediated beta- cell destruction and which changes may be primary and which may be secondary to this process. This part, in Fig. 4, Panel 4, referred to as bioinformatics, now a day involves computer-assisted searches of information e.g. with the aim to group the proteins according to function and pathways. Examples of this are given below. Identification of proteins and protein-pathways of primary interest for further functional characterization also involve genetic analyses, e.g. does the gene encoding the protein map to a chromosomal region demonstrating linkage to T1DM. The final step in the experimental design is to characterize the selected proteins/protein modifications for the putative involvement in cytokine mediated beta-cell destruction and T1DM by functional and further genetic analysis. This involves cloning and recombinant expression of the protein in beta-cell lines to elucidate if the protein influences the known effect of cytokines. If so, further studies in transgenic mice, including the animal model of diabetes, the nonobese diabetic (NOD) mouse, either by knock-out or over-expression of the gene of interest in the beta-cells under control of an insulin-promoter. Others and we have demonstrated the value of this method by overexpressing protective proteins in beta-cell lines result- 

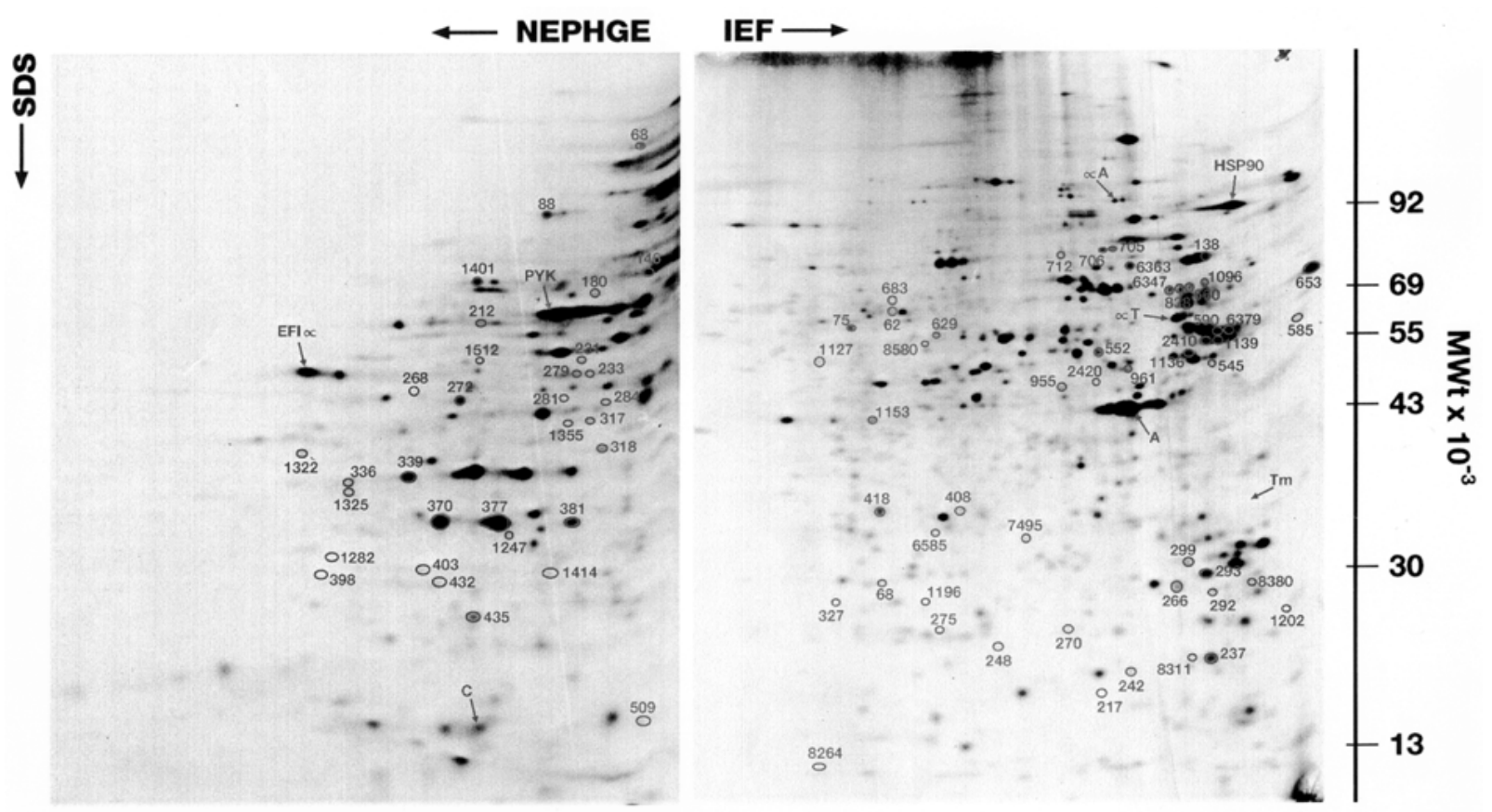

Fig. 5. Fluorograph of a 2-dimensional gel of rat islets of Langerhans. The proteins marked represent proteins changing expression level in response to IL-1 $\beta$ exposure. IEF gel ( $\mathrm{pH} 3.5-7)$ on the right side; NEPHGE gel (pH 6.5-10.5) on the left side.

ing in protection of the cells against subsequent cytokine exposure [24,38,39]. The genetic analyses further may involve screening for polymorphisms of the gene, tests for disease association and linkage, and possible functional significance of identified gene variations. We have previously demonstrated the value of this approach [40-43].

\section{In vitro analysis of il-1 $\beta$ exposed rat islets}

Using the experimental design detailed above, we set out to produce a database of rat islet proteins containing about 2,200 protein spots characterized by Mw and pI $[34,35]$. This demonstrated that IL- $1 \beta$ exposure of the islets in culture resulted in reproducible and statistically significant modulation of protein expression levels or de novo synthesis of 105 of these proteins. Fiftytwo proteins were up-regulated, 47 down-regulated and 6 synthesized de novo [35] (Fig. 5).

Subsequently, we have attempted to positively identify these 105 proteins in order to better understand beta-cell destruction and T1DM at the molecular level, since this would provide the first larger scale assessment of IL- $1 \beta$-mediated beta-cell damaging processes at the protein level.
A success rate of approximately $60 \%$ for positive identifications was obtained in this study [37].

Most identified proteins could be assigned classes according to their known or putative functions: i) energy transduction and redox potentials; ii) glycolytic pathway; iii) protein synthesis, chaperones, and protein folding; and iv) signal transduction, regulation, differentiation, and apoptosis [37]. These findings strongly support the hypothesis that that islet exposure to cytokines induces a complex pattern in beta-cells comprising protective (e.g. up regulation of stress proteins) as well as deleterious (e.g. iNOS induction and NO production) events.

The overall picture is complex and reflects the range of cellular responses to the cytokine challenge. It is not known which protein changes should be considered 'primary' or 'secondary' in importance in time and in sequence. Furthermore, it has been shown that IL- $1 \beta$ may induce both NO dependent as well as NO independent beta-cell impairment $[34,44]$. To further elucidate this, we have recently demonstrated that of the 105 protein spots, which changed expression levels in response to IL-1 $\beta$ [35], only 23 were dependent on NO production [36]. In addition, the effect of the chemical NO donor S-nitroglutathione (GSNO) on protein expression was addressed, demonstrating changes in 


\section{In vitro analysis (rat and human islets)}
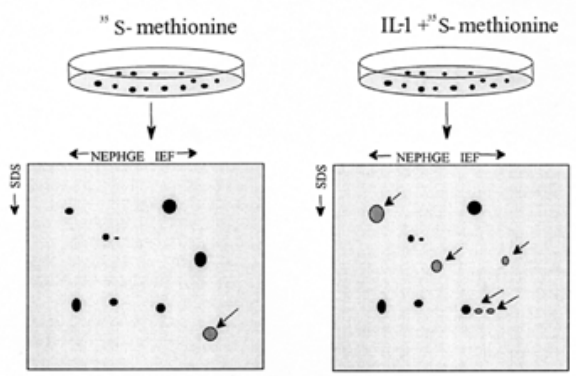

\section{In vivo analysis}
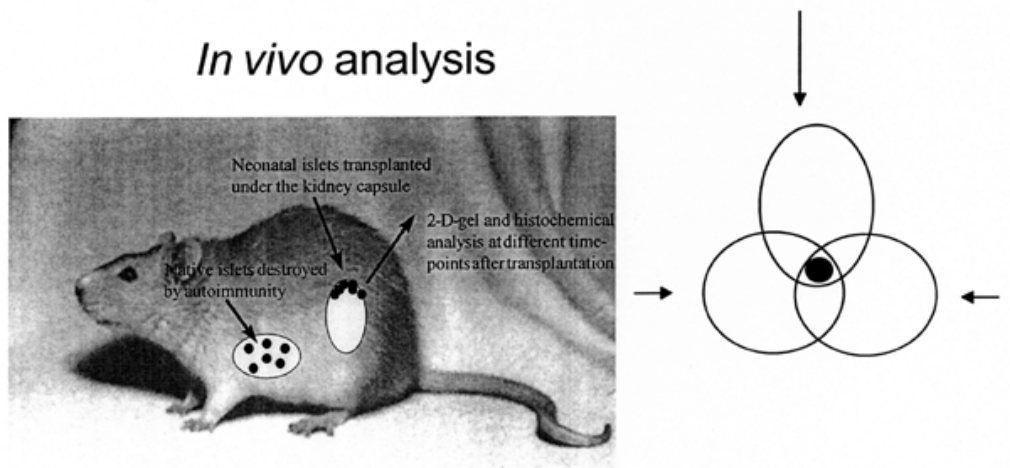

\section{$\beta$-cell maturation -model}

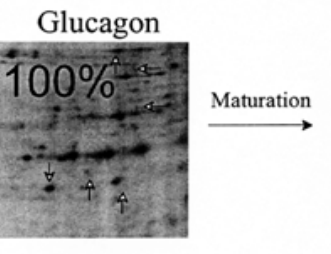

Insulin
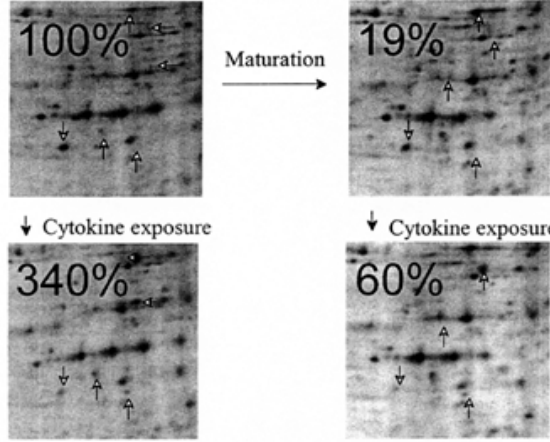

$\downarrow$ Cytokine exposure

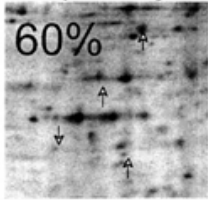

Fig. 6. Extended proteome approach in the study of Type 1 diabetes.

expression levels of 19 islet proteins, which were not identified by MS [36]. This suggests that the majority of protein changes observed in rat islets exposed to IL$1 \beta$ are independent of NO production. It is not clear whether the plethora of changes observed is secondary to the early effects of changes in just one or a few proteins, e.g. involved in mitochondrial energy generation. We are presently finalizing our analysis of isolated human islets exposed to IL- $1 \beta$ alone or IL- $1 \beta$ in combination with IFN $\gamma$ and $\mathrm{TNF} \alpha$. The data obtained in this study are even more complex, but the picture emerging show that the same pathways as in the IL- $1 \beta$ exposed rat islets are affected.

\section{In vivo analysis of IL-1 $\beta$ exposed rat islets}

Whereas the experimental design and data presented above was designed to characterize the proteins involved in cytokine responses in vitro, we have applied proteome-based strategies also to address the influence of immune mediators on beta-cell destruction in vivo during disease progression using an animal model of
T1DM, the inbred diabetes prone BioBreeding (BB) rat $[45,46]$. The overall aim of this extended proteome approach (Fig. 6) is to include and combine information from different experimental procedures with the same focus to narrow down the number of proteins to be functionally characterized for their potential involvement in T1DM development. In this model, syngeneic islets, isolated from newborn rats are transplanted under the kidney-capsule of 30 days old BB rats (time of weaning), approximately 7 weeks before the onset of disease (Fig. 6). Beta-cells of the syngeneic islets transplanted under the kidney capsule are exposed to the same immunological events (e.g. infiltration with macrophages, T- and B-lymphocytes) as the host islets of the pancreas, and presumably they are destroyed in a process identical to that in the host islets. At different time points before disease onset, at the day of onset of disease and in the few animals escaping from diabetes, the transplanted islets are excised and used for proteome as well as histochemical analyses. This allows us to follow the changes in protein expression occurring in the islets until the onset of diabetes. Furthermore, analyses of the islets from the rats that es- 
cape diabetes development, may also give valuable information. This complex set of data is presently being completed, and several of the proteins and pathways found in the in vitro experiments are also identified in the in vivo situation.

\section{A model of beta-cell maturation}

An additional component in our extended proteome approach, is the use of a pluripotent cell line, which dependent of culture condition may mature from a glucagon-producing pre-beta-cell phenotype into an insulin producing beta-cell phenotype [47]. We have recently demonstrated that this maturation is accompanied by an acquired sensitivity to IL- $1 \beta$ [22]. Therefore, this cell-system is useful for characterization of the mechanisms responsible for cytokine sensitivity. Proteome analysis is presently performed of the IL- $1 \beta$ non-sensitive (pre-beta-cell phenotype) vs. the sensitive beta-cell phenotype in the presence or absence of cytokines (Fig. 6). As an example of a preliminary finding from this analysis, one spot was reduced fivefold in expression level following maturation into the cytokine sensitive beta-cell phenotype. After exposure to IL-1 $\beta$, the spot was up-regulated in both phenotypes by a factor of approximately 3.5. This suggests that the protein could represent a scavenger protein involved in scavenging the IL- $1 \beta$ induced toxic FR. Insufficient up-regulation of a scavenger protein in the beta-cell phenotype may result in its destruction. However, at present we do not know the identity of this protein.

\section{Perspective in proteome analysis}

As described above the use of proteome analysis in characterization of processes involved in or responsible for disease progression is a valuable and a rapidly developing area. One major advantage is that it allows analysis of proteins both in their native and modified form. This is in contrast to the rapidly developing transcriptome approach, focusing on the gene-expression profile (the steady state mRNA expression, Fig. 7), where modifications of proteins are not apparent from the mRNA. Thus, whereas the transcriptome technique clearly complements the proteome analysis $[48,49]$, it does not provide all the information obtained by proteome analysis. It is well known that several cellular processes are dependent on posttranslational modifications of an already existing, readily available protein pool in the cell. This includes phosphorylation, glycosylation, sulphatation or cleavage of precursor sequences as they can determine activity, stability, localization and turnover. The ability to detect the same protein in several different spots on a 2D-gel makes it possible to obtain information about potential posttranslational modifications. Whereas this "state of the art" technology for identifying the posttranslational modifications is rapidly developing, it is at present not easily included in the experimental strategies outlined above. A limitation of proteome analysis is the inability to separate all the proteins, even on a large gel. In line with the increasing sensitivity of the MS-identification, this becomes an increasing problem, since contamination with closely neighboring or partly overlapping proteins may result in identification of more than one protein in the same spot. Since MS is not quantitative, it is not possible to determine which protein is the one of changed expression without further analysis, e.g. Western blotting with specific antibodies. This problem may, however, be solved with the use of so called zoom-gels making it possible to look at only small parts of the pI or Mw area in separate gels, and finally combine the information form the different gels, obtaining an increased separation of the protein-spots. Indeed the area is in rapid progression and continued development and what is a bottleneck today may be easily solved tomorrow.

\section{Conclusion}

The use of proteome analysis may elucidate relevant mechanisms for beta-cell death after cytokine exposure. Whether IL- $1 \beta$ induced changes in protein expression levels in rat islets in vitro will also reflect pathogenically important changes in beta-cells in rats spontaneously developing T1DM remains to be determined. Preliminary observations using 2D-gel studies of excised syngeneic islet transplants from different time points posttransplantation in BB-DP rats [46] suggest that the approach will be useful for studies of T1DM pathogenesis in vivo. Interestingly, the exquisite beta-cell sensitivity to cytokine toxicity may be an acquired trait developed during beta-cell maturation [22].

We believe that data obtained by the methodology presented here alone or in combination with e.g. genetic studies, transcriptome analyses and studies of candidate genes e.g. identified through the Copenhagen model (Fig. 8) will lead us to a detailed and complex picture of the molecular processes producing beta-cell 


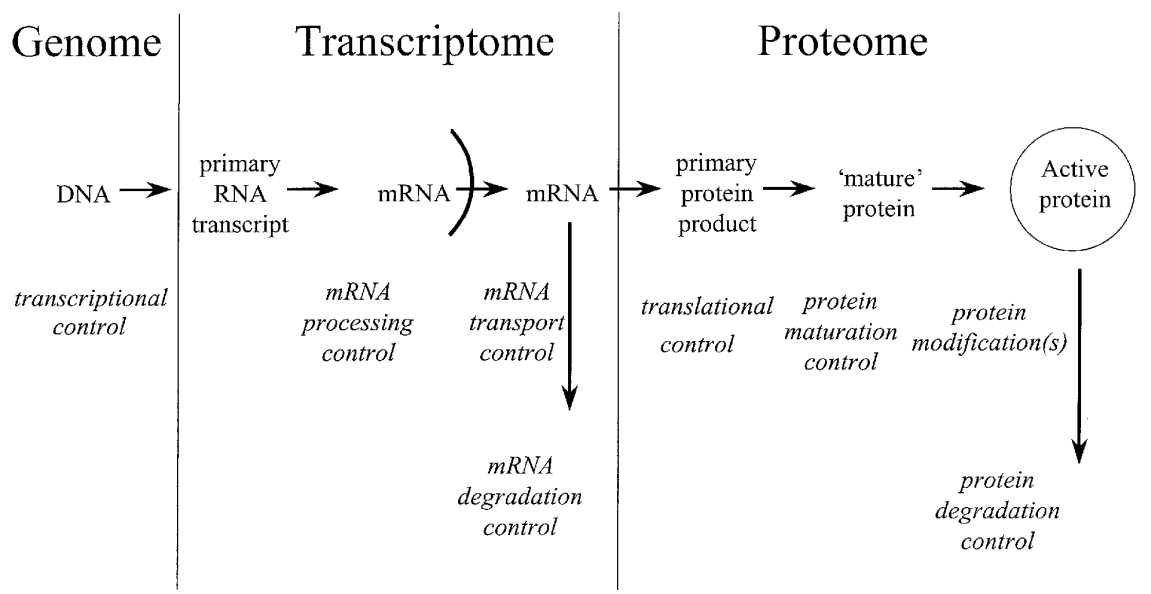

Fig. 7. Approaches in Type 1 diabetes research. Research addressing mechanisms involved in cytokine-mediated beta-cell destruction may be divided in three areas, focusing on the genome, the transcriptome or the proteome as detailed in the text.

\section{Genetic approach}

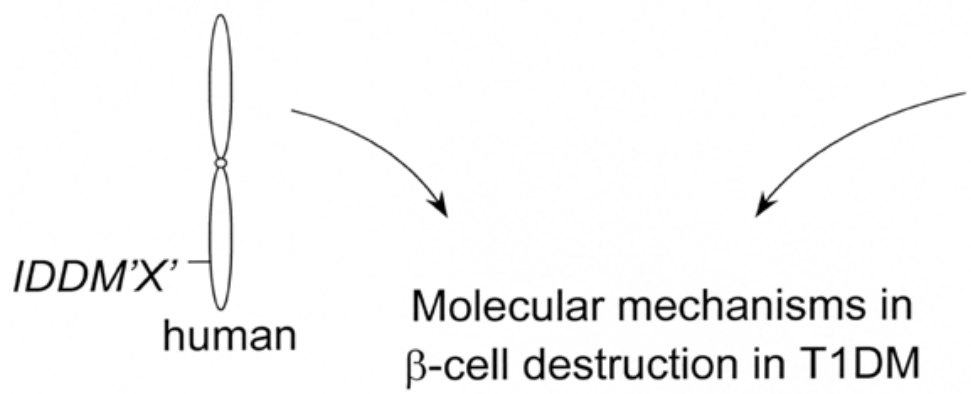

\section{Candidate approach}

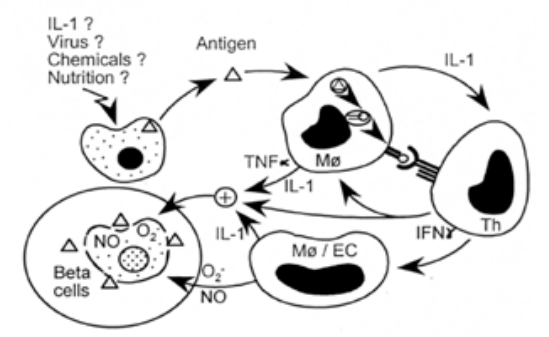

Proteome approach

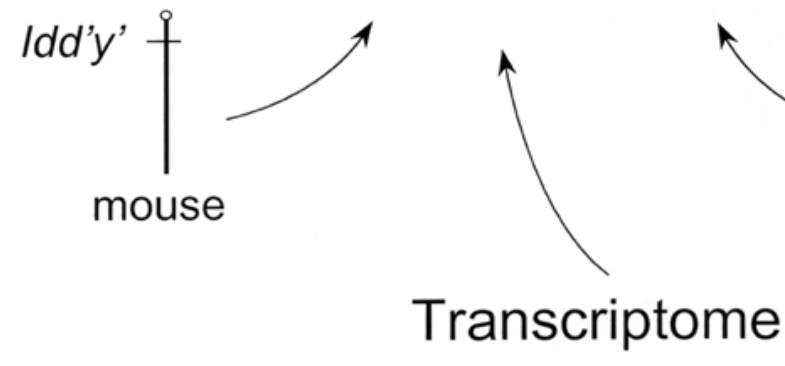

$\mathrm{pl} \rightarrow$

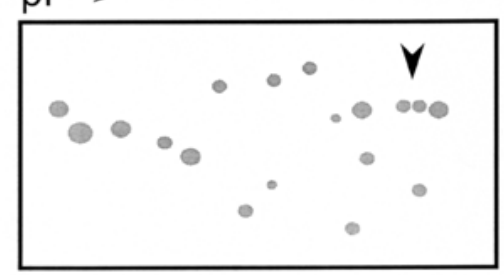

2-D gel

Fig. 8. Identification of molecular mechanisms in beta-cell destruction and Type 1 diabetes. A combined approach taken advantages from genomics, proteomics and biochemical studies is most likely to reveal the molecular mechanisms involved in the beta-cell destruction.

destruction in vitro. Although the picture is complicated and far from complete we are looking at the ailing beta-cell through a new window and the challenge now is to learn to fully understand what we see. Further proteome and transcriptome analyses may eventually complete the picture. The long-term perspective of this is the development of new and specific intervention modalities in beta-cell destruction in T1DM. Making the beta-cell more resistant to immunological mediators may increase the survival time of transplanted islets or engineered beta-cells with reduced immunosuppressive modalities in treatment of T1DM patients, and potentially prevent the ongoing beta-cell destruction in predisposed individuals. 


\section{References}

[1] M. Karvonen, M. Viik-Kajander and E. Moltchanova et al., Incidence of childhood Type 1 diabetes worldwide, Diabetes Care 23 (2000), 1516-1526.

[2] T. Lorenzen, F. Pociot, P. Hougaard and J. Nerup, Long-term risk of IDDM in first-degree relatives of patients with IDDM, Diabetologia 37 (1994), 321-327.

[3] A.G. Moelbak, B. Christau, B. Marner, K. Borch-Johnsen and J. Nerup, Incidence of insulin-dependent diabetes mellitus in age groups over 30 years in Denmark, Diabetic Med 11 (1994), 650-655.

[4] J. Nerup, P. Platz and O.O. Andersen et al., HLA antigens and diabetes mellitus, Lancet 2 (1974), 864-866.

[5] F. Pociot, Insulin-dependent diabetes mellitus - a polygenic disorder? Dan Med Bull (Thesis) 43 (1996), 216-248.

[6] J.-X. She, Susceptibility to Type 1 diabetes: HLA-DQ and DR revisited, Immunol Today 17 (1996), 323-329.

[7] L. Hashimoto, C. Habita and J. Beressi et al., Genetic mapping of a susceptibility locus for insulin-dependent mellitus on chromosome 11q, Nature 371 (1994), 161-164.

[8] J.L. Davies, Y. Kawaguchi and S.T. Bennett et al., A genomewide search for human susceptibility genes, Nature 371 (1994), 130-136.

[9] P. Concannon, K. Gogolinewens and D. Hinds et al., A secondgeneration screen of the human genome for susceptibility to insulin-dependent diabetes-mellitus, Nature Genet 19 (1998), 292-296.

[10] C. Mein, L. Esposito and M. Dunn et al., A search for type1 diabetes susceptibility genes in families from the United Kingdom, Nature Genet 19 (1998), 297-300.

[11] H. Gerstein, Cow's milk exposure and Type 1 diabetes, Diabetes Care 17 (1994), 13-19.

[12] G. Dahlquist, Environmental risk factors in human Type 1 diabetes - an epidemiological perspective, Diabetes Metab Rev 11 (1995), 37-46.

[13] H.S. Jun and J.W. Yoon, The role of viruses in Type 1 diabetes: two distinct cellular and molecular pathogenic mechanisms of virus-induced diabetes in animals, Diabetologia 44 (2001), 271-285.

[14] T. Mandrup-Poulsen, K. Bendtzen, J.H. Nielsen, G. Bendixen and J. Nerup, Cytokines cause functional and structural damage to isolated islets of Langerhans, Allergy 40 (1985), 424429.

[15] T. Mandrup-Poulsen, K. Bendtzen and J. Nerup et al., Affinity purified human interleukin 1 is cytotoxic to isolated islets of Langerhans, Diabetologia 29 (1986), 63-67.

[16] K. Bendtzen, T. Mandrup-Poulsen, J. Nerup, J.H. Nielsen and C. Dinarello, Cytotoxicity of human pI 7 interleukin-1 for pancreatic islets of Langerhans, Science 232 (1986), 15451547.

[17] J. Nerup, T. Mandrup-Poulsen and J. Mølvig et al., Mechanisms of pancreatic beta-cell destruction in Type 1 diabetes, Diabetes Care 11(1) (1988), 16-23.

[18] W. Suarez-Pinzon, K. Strynadka, R. Schulz and A. Rabinowitch, Mechanisms of cytokine-induced destruction of rat insulinoma-cells: The role of nitric oxide, Endocrinology 134 (1994), 1006-1010.

[19] D.L. Eizirik, D.G. Pipeleers and Z. Ling et al., Major species differences between humans and rodents in the susceptibility to pancreatic $\beta$-cell injury, Proc Natl Acad Sci USA 91 (1994), 9253-9256.
[20] J.A. Corbett and M.L. McDaniel, Intraislet release of interleukin 1 inhibits $\beta$ cell expression of inducible nitric oxide synthase, J Exp Med 181 (1995), 559-568.

[21] M. Di Matteo, A. Loweth and S. Thomas et al., Superoxide, nitric oxide, peroxynitrite and cytokine combinaitons all causes functional impairment and morphological changes in rat islets of Langerhans and insulin secreting cell lines, but dictate cell death by different mechanisms, Apoptosis 2 (1997), 164-177.

[22] K. Nielsen, A.E. Karlsen and M. Deckert et al., Beta-cell maturation leads to in vitro sensitivity to cytotoxins, Diabetes 48 (1999), 2324-2332.

[23] A.E. Karlsen, D. Pavlovic and K. Nielsen et al., Interferongamma induces interleukin-1 converting enzyme expression in pancreatic islets by an interferon regulatory factor-1dependent mechanism, J Clin Endocrinol Metabol 85 (2000), 830-836.

[24] S. Lortz, M. Tiedge and T. Nachtwey et al., Protection of insulin-producing RINm5F cells against cytokine-mediated toxicity through overexpression of antioxidant enzymes, Diabetes 49 (2000), 1123-1130.

[25] A.E. Karlsen, H.U. Andersen and H. Vissing et al., Cloning and expression of cytokine inducible nitric oxide synthase cDNA from rat islets of Langerhans, Diabetes 44 (1995), 753-758.

[26] C.A. Delaney, D. Pavlovic, A. Hoorens, D.G. Pipeleers and D.L. Eizirik, Cytokines induce deoxyribonucleic acid strand breaks and apoptosis in human pancreatic islet cells, Endocrinology 138 (1997), 2610-2614.

[27] A.E. Karlsen and T. Dyrberg, Molecular mimicry between non-self, modified self and self in autoimmunity, Sem Immunol 10 (1998), 25-34.

[28] J. Nerup, T. Mandrup-Poulsen and S. Helqvist et al., On the pathogenesis of IDDM, Diabetologia 37(2) (1994), S82-S89.

[29] T. Mandrup-Poulsen, The role of interleukin-1 in the pathogenesis of insulin-dependent diabetes mellitus, Diabetologia 39 (1996), 1005-1029.

[30] B. Freiesleben-De Blasio, P. Bak, F. Pociot, A.E. Karlsen and J. Nerup, Onset of Type 1 diabetes: a dynamical instability, Diabetes 48 (1999), 1677-1685.

[31] W.A. Hagopian and A. Lernmark, Islet cell autoantibodies (1996), 441-448.

[32] W.E. Winter, The use of islet autoantibody markers in the prediction of autoimmune Type 1 diabetes, Clin Immunol Newsletter 19 (1999), 25-39.

[33] S.P. Gygi, Y. Rochon, B.R. Franza and R. Aebersold, Correlation between protein and mRNA abundance in yeast, Mol Cell Biol 19 (1999), 1720-1730.

[34] H.U. Andersen, P.M. Larsen and S.J. Fey et al., Twodimentionel gel electrophoresis of rat islets proteins: Interleukin-1 beta induced changes in protein expression are reduced by L-argenine depletion and nicotinamide, Diabetes 44 (1995), 400-407.

[35] H.U. Andersen, S.J. Fey and P. Mose Larsen et al., Interleukin1beta induced changes in the protein expression of rat islets, Electrophoresis 18 (1997), 2091-2103.

[36] N. John, H. Andersen and S. Fey et al., Cytokine or chemicallyderived nitric oxide alters the expression of proteins detected by two-dimensional gel electrophoresis in neonatal rat islets of Langerhans, Diabetes 49 (2000), 1819-1829.

[37] P.M. Larsen, S.J. Fey and M.R. Larsen et al., Proteome analysis of interleukin-1 beta-induced changes in protein expression in rat islets of Langerhans, Diabetes 50 (2001), 1056-1063.

[38] A. Rabinovitch, W. Suarez-Pinzon and K. Strynadka et al., Transfection of human pancreatic islets with an anti- apop- 
totic gene (bcl-2) protects beta-cells from cytokine- induced destruction, Diabetes 48 (1999), 1223-1229.

[39] S. Grey, M. Avelo, W. Hasenkamp, F. Bach and C. Ferran, A20 inhibits cytokine-induced apoptosis and nuclear factor kB-dependent gene activation in islets, J Exp Med 190 (1999), 1135-1145.

[40] F. Pociot, J. Mølvig, L. Wogensen, H. Worsaae and J. Nerup, A TaqI polymorphism in the human interleukin- $1 \beta$ (IL-1 $\beta$ ) gene correlates with IL-1 $\beta$ secretion in vitro, Eur J Clin Invest 22 (1992), 396-402.

[41] F. Pociot, A.G. Wilson, J. Nerup and G.W. Duff, No independent association between a TNFa promoter region polymorphism and insulin-dependent diabetes mellitus (IDDM), Eur J Immunol 23 (1993), 3043-3049.

[42] O.P. Kristiansen, M. Zamani and J. Johannesen et al., Linkage and association between a CD4 gene polymorphism and IDDM in Danish IDDM patients, Diabetes 47 (1997), 281283.

[43] R. Bergholdt, Z.M. Larsen and N.A. Andersen et al., Characterization of new polymorphisms in the 5' UTR of the human interleukin-1 receptor Type 1 (IL1R1) gene: linkage to Type 1 diabetes and correlation to IL-1RI plasma level, Genes Immunol 1 (2000), 495-500.
[44] D.L. Eizirik, M. Flodström, A.E. Karlsen and N. Welsh, The harmony of the spheres: inducible nitric oxide synthase and related genes in pancreatic beta cells, Diabetologia 39 (1996), 875-890.

[45] U. Christensen, T. Sparre and A. Cooke et al., Syngeneic islet transplantation in prediabetic BB-DP rats - a synchronized model for studying beta-cell destruction during the development of IDDM, Autoimmunity 28 (1998), 91-107.

[46] U.B. Christensen, P.M. Larsen and S.J. Fey et al., Islet protein expression changes during diabetes development in islet syngrafts in BB-DP rats and during rejection of BB-DP islet allografts, Autoimmunity 32 (2000), 1-15.

[47] O.D. Madsen, C. Karlsen and P. Serup et al., in: Insulin secretion and pancreatic B-cell research, P.R. Flatt and S. Lenzen, eds, Smith-Gordon, UK, 1994, pp. 61-68.

[48] K. Rieneck, L. Bovin and K. Josefsen et al., Masssive parallel gene expression profiling of RINm5F pancreatic islet $\beta$-cells stimulated with interleukin-1 $\beta$, APMIS 108 (2000), 855-872.

[49] A. Cardozo, M. Kruhoeffer, R. Leeman, T. Orntoft and D. Eizirik, Identification of novel cytokine-induced genes in pancreatic beta-cells by high density oligonucleotide arrays, Diabetes 50 (2001), 909-920. 


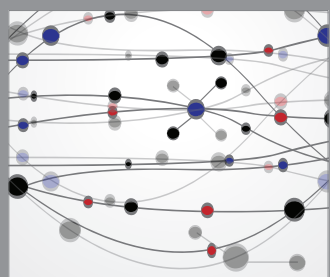

The Scientific World Journal
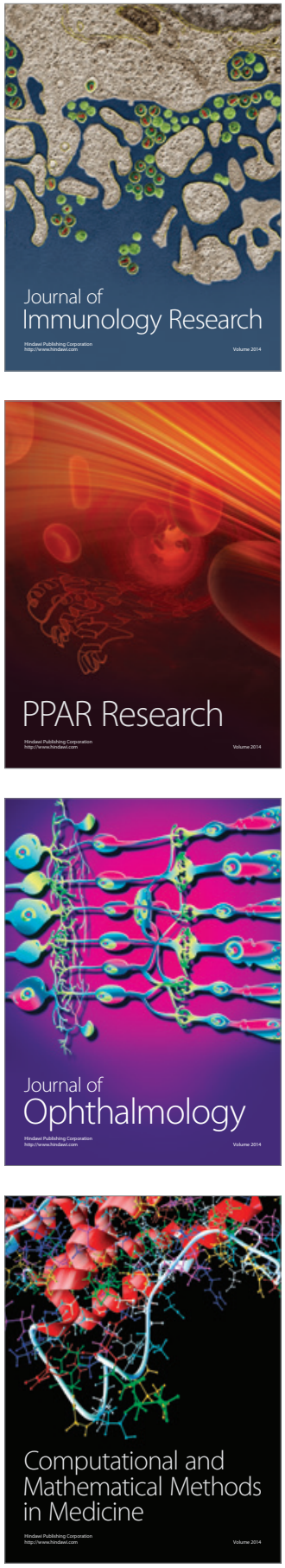

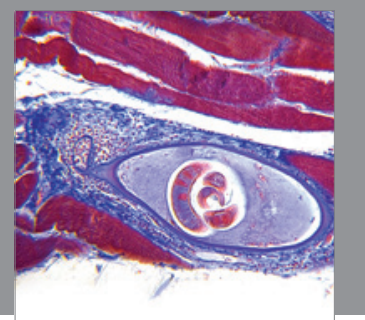

Gastroenterology

Research and Practice
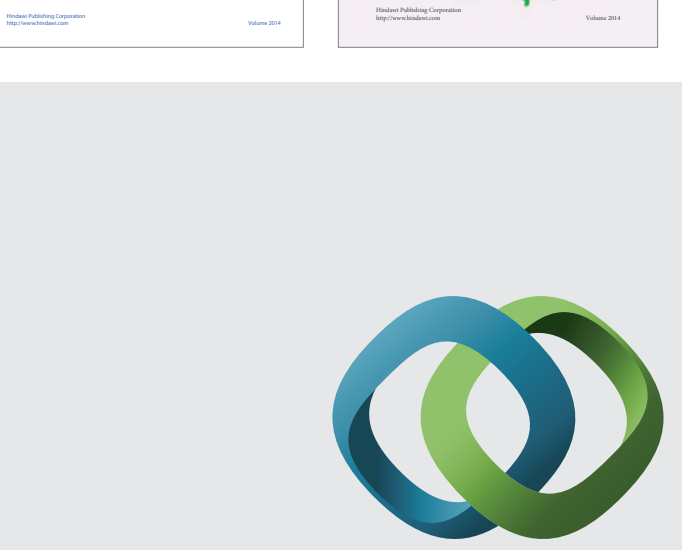

\section{Hindawi}

Submit your manuscripts at

http://www.hindawi.com
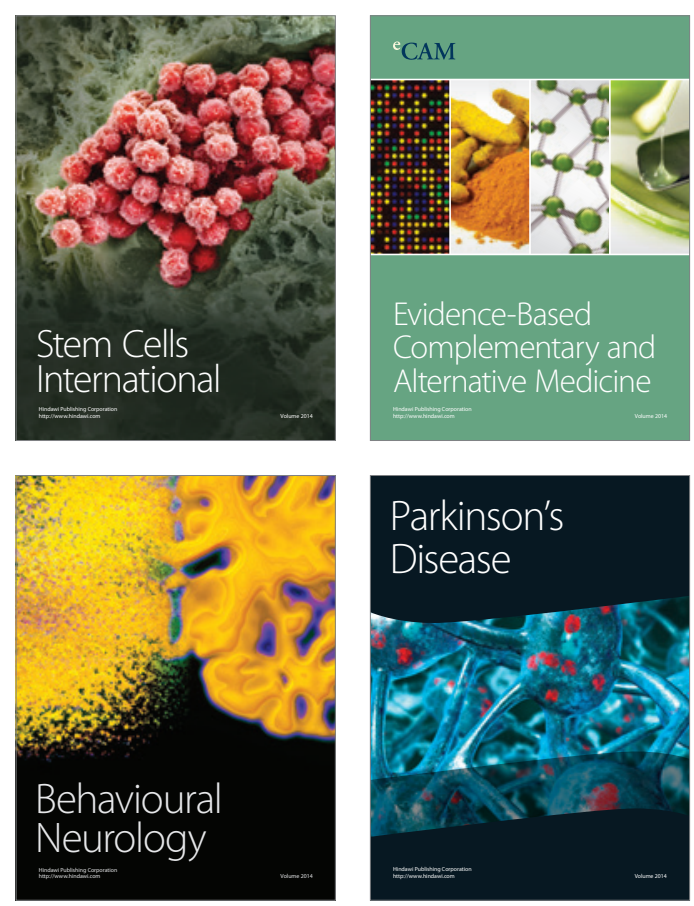

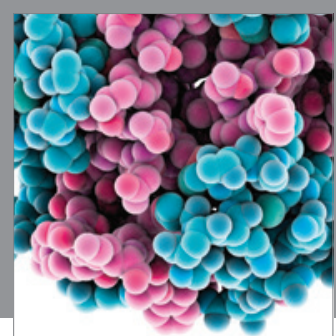

Journal of
Diabetes Research

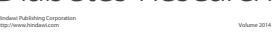

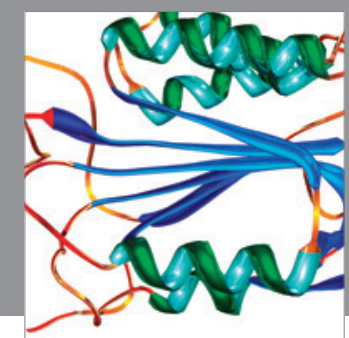

Disease Markers
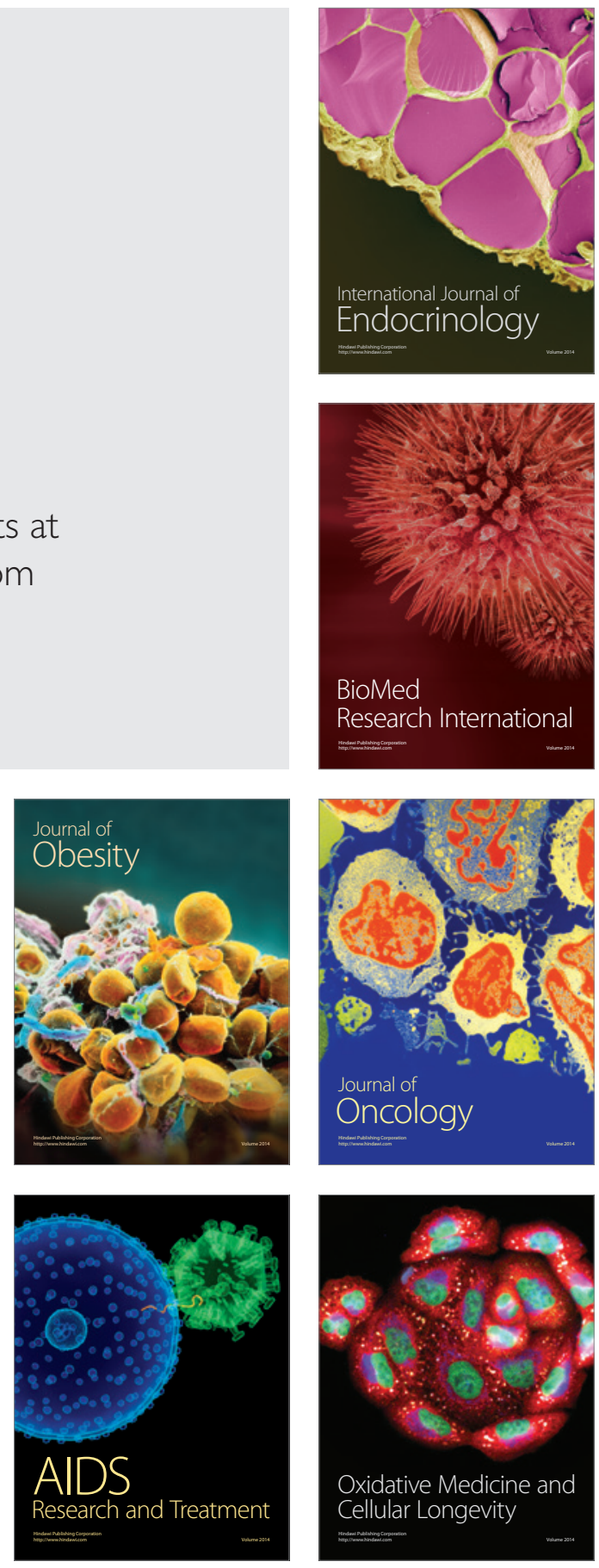